\title{
Insegnare l'italiano per fini specifici: motivazione e apprendimento cooperativo
}

\author{
Angela Ferioli
}

\begin{abstract}
The purpose of this communication is to situate cooperative language learning and teaching in the sphere of foreign language education, specially of the Italian as second language to students of a M anagement School. The concepts of collaborative or cooperative learning have emerged in the last fifteen years, incorporating principles of learner-centrededness into programs. With regard to this point, the aim is to motivate the students of technical schools or colleges to learn the L2 for their future jobs. O ne of the basic teaching techniques associated with cognitive theory is based on activities related to the learners' subject specialization. Yet, this is not enough to motivate young students to improve the four traditional skills in the target language. So, in the classroom of a foreign language for specific purposes, teacher can link the various methodological approaches with cooperative learning in small groups, to increase the awareness about learning, and about language, and to implement an integrative motivation.

The collaborative efforts to work together in the classroom, with the students organized in structured teams, involve and encourage to develop metacommunicative as well as communicative skills.
\end{abstract}

\section{Introduzione}

Scopo di questa comunicazione è la proposta dell'apprendimento cooperativo (AC) come approccio pedagogico per l'italiano diretto a studenti di Economia Aziendale, vale a dire per l'italiano come microlingua del commercio e dell'economia.

Prima di tutto si giustificherà l'adozione di questa metodologia dal punto di vista teorico, attraverso una breve riflessione sugli aggiornamenti in glottodidattica, nel cui discorso I'AC può inserirsi come elemento integrativo. In un secondo tempo si tenterà di chiarire termini e concetti, indicando alcuni riferimenti pratici per organizzare l'impostazione didattica in classe.

\section{Come insegnare I'IFS, ovvero dal processo al prodotto}

II processo di apprendimento può essere paragonato a un lungo viaggio. La metafora è proposta dai linguisti britannici $\mathrm{H}$ utchinson e $\mathrm{W}$ aters, $\mathrm{i}$ quali coraggiosamente si chiedono perché l'alunno di una facoltà non letteraria 
dovrebbe iniziare un percorso in territorio sconosciuto e forse ostile. $E$ se non avesse voglia di cominciare? 0 ppure, se non volesse continuare il viaggio già iniziato e decidere di non arrivare a destinazione? Siamo proprio sicuri che gli alunni di Economia siano motivati a leggere in italiano un bilancio consolidato? In realtà non c'è nulla di garantito, nemmeno per l'inglese, figuriamoci per l'italiano! Queste considerazioni ci conducono all'analisi dei bisogni, individuando una distinzione tra esigenze strumentali e bisogni integrativi o formativi (H utchinson-W aters, 1987; Porcelli, 1992; Freddi, 1994). I primi li troviamo negli apprendenti adulti, già inseriti nel mondo dell'impresa il cui approccio verso la lingua per fini specifici (LFS) ha uno scopo cosciente, vale a dire il raggiungimento di obiettivi professionali concreti (comunicare con clienti e fornitori, ecc.). T uttavia, questo tipo di esigenze sono ancora labili negli studenti di Economia di una fascia di età compresa tra i 18 e i 24 anni, i quali di solito mostrano una tiepida motivazione verso le lingue estere. Infatti, durante i corsi di LFS essi dovranno affrontare una serie di obiettivi linguistici che intrinsecamente potrebbero considerare noiosi e demotivanti.

I nostri alunni studiano la $L 2$ entro le pareti scolastiche, dove l'insegnante è il veicolo principale della conoscenza linguistica. T uttavia, il rapporto insegnante-alunno non è il solo tramite per far praticare l'uso della lingua. Se il contesto di apprendimento è la classe, gli alunni possono imparare a condividere le informazioni tra di loro, cioè a integrarsi in quel contesto particolare, visto che per il momento è l'unico disponibile per praticare la lingua. N ulla di nuovo, si può obiettare, eppure in un corso di italiano per fini specifici (IFS) la cooperazione tra gli alunni presenta una certa complessità, anche per le caratteristiche intrinseche della microlingua.

Fin dal 1971, R. Titone aveva segnalato questi problemi psicologici, sviluppati anche da Gardner (1972) e da Lambert (1985). Essi individuarono come bisogni integrativi la somma delle diverse variabili che intervengono nella volontà d'integrarsi, appunto, in una classe di $L 2$ : variabili sociali, l'età o il diverso sviluppo cognitivo-linguistico dei discenti, pregiudizi e disponibilità verso la comunità che parla la lingua meta 0 , come nel nostro caso, che esprime una determinata cultura d'impresa. A tutto questo bisogna aggiungere la disponibilità di lavorare efficacemente con i propri compagni.

L'apprendimento di una L 2 è una sorta di riapprendimento linguistico e, inoltre, comprende un'estensione 0 una rieducazione nelle abilità comunicative di ruoli sociali specifici. Tradotto in termini di curricolarità potremmo considerare le fasi dell'unità didattica (UD) in questo modo:

1. Elaborazione dei materiali linguistici senza perdere mai di vista l'obiettivo strumentale della microlingua: I'alunno impara per lavorare in azienda. Diventa necessaria una particolare attenzione nelle scelte dei contenuti del programma, prendendo atto della stretta collaborazione tra linguaggio settoriale e lingua standard; 
2. Ie operazioni didattiche devono tentare di risolvere il problema di come passare dall'uso alla regola. Titone (1991 e 1992) e Balboni suggeriscono che per stimolare la consapevolezza linguistica, o Language Awareness, una strategia di apprendimento dovrebbe coinvolgere non solo la componente cognitiva (per es. come fissare i dati in memoria), ma anche le abilità sociali che sono alla base della componente affettiva (per es. capacità di ascoltare gli altri; rispetto delle differenze);

3. Ie tecniche didattiche da impiegare dovrebbero orientarsi verso una collaborazione tra abilità comunicative e metacomunicative.

II modello che ha messo a fuoco i presupposti dei punti 2 e 3 , sforzandosi di offrire una risposta applicata, pensiamo sia quello elaborato da Kagan (1993) per l'apprendimento cooperativo.

\section{Abilità e apprendimento cooperativo}

Integrando gli approcci comunicativi e cognitivi, I'AC affronta l'esigenza e la responsabilità di offrire nuovi contenuti educativi, con la proposta di un amplio campo di strutture d'apprendimento. L'insegnante, dunque, dovrebbe organizzare curricoli orientati alla formazione di sistemi di padronanza tali che l'allievo possa riutilizzarli non solo nel contesto scolastico, ma soprattutto in quello professionale.

I canali di trasmissione della lingua interferiscono sull'acquisizione, sul suo uso e sulle scelte metodologiche per insegnarla. L'impatto della società informativa odierna è preso in considerazione da Freddi (1994), Voghera (1992) e Kagan (1993), per quanto riguarda il ruolo della memoria in que sta nostra società interattiva e informatizzata. Se i computer hanno una memoria di ferro, che senso ha per l'apprendente archiviare dati in memoria in modo stereotipato e automatico? I nostri alunni studiano le applicazioni strategiche e operative delle tecnologie informatiche nelle varie aree aziendali. H anno il compito di acquisire una conoscenza corretta dei sistemi di raccolta, archiviazione, elaborazione e trasmissione dei dati e delle informazioni. Per chi opera in ambito economico, prendere decisioni corrette in tempi molto brevi non lascia spazio a vecchie impostazioni di archiviazione dei dati. L'insegnamento, in generale, dovrebbe complementare la conoscenza dei contenuti, cioè la quantità di ciò che s'impara, con le abilità orientate a identificare, classificare, elaborare, sintetizzare per condividire la conoscenza. Tuttavia, per rendere effettiva la comunicazione è necessario riuscire a comunicare. Sembra una banalità, ma siamo sicuri che tutti gli alunni, o almeno una parte accettabile della classe, possegga le abilità sociali necessarie per lavorare insieme?

Senza una rete informativa efficace, I'abilità di elaborazione dei dati cade nel vuoto e gli alunni non sapranno condividere nulla, né in classe né in azienda. 


\section{Alcuni concetti chiave}

$\mathrm{N}$ on consideriamo una novità formare a volte dei gruppi di lavoro nella nostra classe. Tuttavia, i gruppi di lavoro includono studenti che lavorano insieme, senza comprendere necessariamente i concetti chiave dell'AC.

$L ' A C$ si riferisce a un insieme di istruzioni strategiche che comprendono gli aspetti seguenti:

- organizzazione di squadre di alunni;

- una varietà di attività complesse per costruire la squadra;

- sviluppo in classe di un'atmosfera collaborativa che comprende una varietà di attività che coinvolgono individuo-gruppo e gruppo-classe;

- un addestramento in ruoli sociali, abilità di comunicazione e abilità di gruppo;

- problemi da risolvere individualmente e poi da verificare tra i compagni di gruppo, prima che siano presentati all'insegnante;

- compiti specifici per squadre: studenti che consultano studenti di altre squadre;

- un sistema speciale di verifica e valutazione che riguarda gli alunni come individui, come squadra e in rapporto a tutta la classe.

Q uesto elenco non vuole essere una specie di litania trionfalistica, per convincere a tutti i costi della bontà dell'AC. Infatti, la messa in atto di qualsiasi strategia glottodidattica dipende anche dalla buona strutturazione del programma. L'applicazione dei punti sopra esposti può essere provata senza dimenticare il buon senso, in modo graduale e sperimentale, puntando, per esempio, sulle strategie di presa di coscienza linguistica.

\section{T eams. Squadre}

\subsection{Cosè una squadra?}

Un gruppo, generalmente, può essere formato da un numero più o meno nume roso di studenti, non ha né deve avere un' identità e la sua durata è al eatoria.

Le squadre dell'AC, invece, devono costruirsi un'identità, attraverso degli esercizi comunicativi, all'inizio del corso. Inoltre, si compongono di quattro membri e devono durare un tempo abbastanza lungo, anche se non un intento quadrimestre 0 un semestre. Sinceramente, la rotazione è uno degli aspetti più difficili da risolvere. Infatti gli alunni si abituano a un gruppo di persone e non vogliono fare altri sforzi per continuare il lavoro con nuovi membri. Tuttavia, l'insegnante dovrebbe dare un tempo limitato alla durata delle squadre, per due motivi:

1. U na squadra formata a caso all'inizio del corso corre il rischio di comprendere solo studenti principianti assoluti, oppure elementi «difficili» dal rendimento basso, o quattro alunni dalla stessa competenza alta, fattori distorsionanti per la collaborazione. 
2. L'addestramento ad accettare nuovi compagni per il lavoro in équipe è una risorsa orientata soprattutto verso il futuro professionale dell'alunno.

II metodo più comune, dunque, per assegnare gli studenti a una squadra è quello dell'eterogeneità. La squadra non omogenea è lo specchio di tutta la classe e deve comprendere, fin dov'è possibile, alunni principianti efalsi principianti, oppure alunni dal rendimento basso, medio e alto.

I livelli eterogenei di rendimento scolastico ottimizzano un insegnamento non gerarchizzato, non cristallizzato nel rapporto alto-basso: professore-alunno. Inoltre, servono anche come aiuto alla direzione della classe numerosa.

\subsection{Come vengono formate le squadre?}

Ci sono vari modi per formare le squadre. Gli alunni possono raggrupparsi per interessi comuni, o anche per amicizia; oppure i membri di una squadra possono unirsi a caso, o è l'insegnante a formare le squadre.

Come si è già detto, le squadre possono anche non essere eterogenee. In questo caso, quali rischi comporta una sel ezione fatta dagli studenti, per formarle? Può verificarsi un rinforzo dello stato gerarchico in classe (i gruppi di non principianti emarginano i principianti), e si producono delle squadre «perdenti». Benchél'elemento competitivo possa considerarsi complementare alle attività collaborative, è consigliabile evitare una drastica polarizzazione dell a classe. In questo caso, è meglio che sial'insegnante a formare delle squadre eterogenee, per poi passare ogni tanto ad altre formate a caso, oppure con un livello di lingua omogeneo.

Per non fare confusione in classe, quando si assegnano gli studenti alle squadre (non dev'essere per forza il primo giorno di lezione), l'insegnante può preparare degli elenchi con i nomi degli alunni di ogni gruppo. In tal modo, in una classe di 27 persone, si preparano 6 elenchi per 6 équipes di quattro alunni + un elenco con una squadra di tre. Si mettono sui banchi tutti gli elenchi con i nomi, in modo che gli studenti sappiano dove andare a sedersi.

\subsection{Perché è più razionale una squadra di quattro persone}

La logica dell'AC si basa sulla partecipazione attiva. In un gruppo di 3 persone, di solito si forma una coppia e un «outsider». A differenza di un gruppo di tre alunni, uno di 4 raddoppia la probabilità di un'ottima contrapposizione linguistica e cognitiva. Infatti, in un gruppo di 3 al unni ci sono tre possibili coppie, mentre in uno di 4 ce ne sono sei.

\subsection{Q ualora non sia possibile formare le squadre di quattro alunni}

In questo caso si possono seguire le seguenti possibilità:

a) uno studente lasciato fuori può inserirsi in una squadra dove può imparare meglio 0 aiutare meglio; 
b) se gli studenti lasciati fuori sono due, scegliere uno studente già assegnato a una squadra e formare una squadra di tre alunni;

c) con tre studenti lasciati fuori, formare una squadra di tre.

\subsection{Più ragazzi che ragazze?}

Spesso in classe ci sono più ragazzi che ragazze, o viceversa. In questo caso, è meglio assegnare 2 ragazzi e 2 ragazze ad ogni squadra e poi formare squadre solo di ragazzi o solo di ragazze. $\dot{E}$ stato osservato che in un équipe di tre ragazzi e una ragazza, questa purtroppo assume un ruolo subalterno, comportandosi da «segretaria del direttore», vale a dire prendendo appunti, ascoltando ma intervenendo poco, ecc. È da rilevare che, globalmente, la maggior parte delle ragazze raggiunge risultati più soddisfacenti dei compagni della stessa età.

\section{Lingua e squadre}

\subsection{Il livello linguistico non è omogeneo}

Q uando si parla di microlingua ci si trova, generalmente, di fronte a studenti che conoscono «l'argomento» e che sono facilitati nella comprensione della lingua proprio dalla familiarità e dall'interesse per esso. Purtroppo, però, una buona parte delle matricole non ha alcuna dimestichezza con la specializzazione che ha scelto. Inoltre, le Facoltà tecniche e scientifiche accolgono studenti provenienti da scuole superiori differenti e forniti da un diseguale bagaglio di conoscenze e abilità metalinguistiche. II calendario delle lezioni non permette l'organizzazione di lezioni a gruppi di diversi livelli. Per quanto riguarda il livello principianti, all'inizio del corso gli studenti possono essere divisi in gruppi omogenei per conoscenza linguistica.

Inoltre, d'accordo con Gotti (1987), il materiale linguistico dovrà essere proposto seguendo un criterio di gradazione. L'insegnante dovrà stabilire quali e quanti elementi del programma dovranno essere insegnati prima e quali dopo.

$\mathrm{N}$ el nostro caso prendiamo in considerazione dapprima gli aspetti più semplici e poi quelli più complessi, con particolare considerazione per gli elementi di interferenza lessicale e linguistica tra italiano, spagnolo, catalano, oppure lessicale per quanto riguarda inglese/italiano.

All'inizio, dunque, dovrebbe essere parlato esclusivamente l'IFS se i contenuti grammaticali e lessicali sono a un livello adeguato per la maggior parte dei gruppi.

\section{Attività, sussidi e contenuti}

Un altro concetto fondamentale di una lezione collaborativa è senz'altro quello di sussidio. Abbiamo tradotto la parola inglese «structure» (proposta 
da Kagan) con «sussidio» per non incorrere in confusioni quando parliamo di strutture della lingua.

Squadra e sussidio sono le basi portanti dell'organizzazione integrata della classe. La squadra ha come obiettivo ordinare l'interazione sociale tra gli alunni. Per integrarsi nel gruppo, e allo stesso tempo raggiungere gli obiettivi fissati dall'UD, essi avranno bisogno di sussidi specifici le cui strategie coinvolgono sia gli aspetti linguistici e metalinguistici, sia quelli comunicativi. Tuttavia, un sussidio dell'AC non è da confondere con un'attività del metodo nozionale-funzionale.

$\mathrm{N}$ el paragrafo che segue cercheremo di chiarire meglio il concetto di sussidio dell'AC.

\subsection{U n sussidio dell'AC è vuoto di contenuti}

II sussidio dell'AC è una cornice vuota di contenuti e può essere usato per affrontare contenuti diversi. La maggior parte delle attività comunicative o di scrittura, sono pensate invece per raggiungere un solo obiettivo a breve termine. Esse possono aiutare in determinati contesti, ma non sono applicabili ad altri. Insomma, molte attività, anche se non tutte, sono di poco uso (per non dire di un solo uso).

Si dovrebbe avere a disposizione una serie di attività da applicare a contenuti diversi per obiettivi diversi. Q uando introduciamo un determinato contenuto all'interno di un sussidio, produciamo un'attività. Se invece si decide di mettere a fuoco obiettivi diversi, nella «cornice» s'inserisce un contenuto diverso, cambiando in tal modo di attività. $\mathrm{N}$ el cambiare sia il sussidio sia il contenuto, produciamo una modalità diversa di apprendimento.

U na didattica riuscita comprende l'abilità di scegliere la miglior combinazione sussidio/contenuti per raggiungere un determinato obiettivo dell'UD .

D escriveremo brevemente alcuni sussidi di padronanza (M astery Structures). Proponiamo quelli che esercitano gli elementi della grammatica o del lessico specialistico quando le attività funzionali si rivelano insufficienti a fissarli in memoria. Infatti, alcuni sussidi sono stati ideati per esercitare in modo interattivo l'elaborazione eil richiamo dei dati in memoria.

Con la struttura «gioco delle carte», che è la più semplice, il contenuto può variare, dipendendo dagli obiettivi prefissati.

Come funziona:

1. O gni squadra si divide in due coppie. L'insegnante prepara una serie di carte che riporteranno da una parte un quesito su un el emento particolare di morfo-sintassi, lessico o semantica. Sul dorso della carta viene scritta la soluzione.

Esempio: parte frontale ESSERE IO

parte dorsale IO SO N O

2. Prima d'iniziare il «gioco» |'insegnante aiuta o invita gli alunni a formulare delle frasi in italiano che servino poi da rinforzo positivo per chi 
esegue correttamente gli esercizi («Brava! Bravissimo!», o altre più divertenti). È fondamentale che un riconoscimento positivo sia dato dai propri compagni di squadra e non solo dall'insegnante. Infatti, esso crea un «buon ambiente» comunicativo, abbassa la soglia dell'ansia, diminuisce il senso del ridicolo e rafforza lo spirito di gruppo.

3. Inizia il gioco: I'alunno A mostra la parte frontale delle carte all'al unno $B$, il quale deve dare la risposta corretta. Se la risposta è corretta B fa un punto e A gli mostra il dorso della carta, congratulandosi con B (che di solito è contentissimo di sentirsi dire che è un genio). Risposta sbagliata: I'alunno A mostra sempre il dorso della carta, cioè la risposta corretta che poi ritorna in fondo al mazzo, per offrire una seconda opportunità a B. Si continua così finché terminano le carte. Scambio di ruoli. $\mathrm{C}$ hi ha vinto il primo round?

Gli alunni $C$ e D eseguono lo stesso esercizio, poi scambiano il mazzo di carte con $A$ e $B$. Verifica dei punteggi.

II momento dell'U D coinvolto è quello della fissazione dei materiali contenuti dal testo-pivot.

Altre applicazioni:

- Tempi verbali, articoli, genere e numero sostantivi, uso delle preposizioni, ecc.

- Recupero di elementi di una lezione precedente. Per esempio, la ricostruzione di sequenze da una conversazione da un'audiocassetta.

- Abbinamento di termini tecnici con le rispettive definizioni.

Q uest'ultimo controllo serve per mettere a fuoco le strategie definitorie, dato che il «saper definire» è una delle sottoabilità più importanti del discorso microlinguistico (Porcelli, 1992). Un corso di LFS deve rivalutare la memoria come mezzo per lavorare anche in una fase intermedia di fissazione e di rielaborazione dei termini economici. $\mathrm{N}$ on sempre è possibile proporre come testipivot dei dialoghi situazionati o letture specifiche. Arriva il momento in cui si deve pur affrontare I'UD «Borsa e Finanza», in cui una delle attività è la decodificazione in L2 di un bilancio aziendale. G eneralmente, gli alunni del quarto anno sanno decodificare senza troppi problemi le voci di bilancio, poiché ne conoscono i concetti finanziari. Per l'insegnante di IFS, dunque, I'obiettivo è ricorrere alle strategie definitorie per passare dal concetto al termine. Attraverso il concetto l'alunno acquisirà il termine «conto economico» che a sua volta metterà in rapporto con «estado de resultados», oppure comprenderà che alle «immobilizzazioni finanziarie» corrisponde «semplicemente» (almeno per lo studente di Economia!) lo stesso concetto di «fijo financiero».

II «gioco delle carte», dunque, è un ottimo sussidio interattivo per acquisire (e non solo memorizzare) questi termini. Da qui l'alunno avanzato può passare a una fase ulteriore, quella della formalizzazione, e tentare la definizione di un grafico, applicando i termini dell'attivo e del passivo. 
Allo stesso tempo, molte attività che derivano dalla ricerca in glottodidattica delle LFS, possono venire integrate dall'organizzazione collaborativa in classe.

Se si preferisce, si possono combinare le attività contestualizzate dell' approccio nozionale-funzionale con le strutture dell'AC. Personalmente, combino i vari approcci e, tuttavia, per certi aspetti grammaticali, comel'uso di preposizioni o di alcune congiunzioni, I'applicazione del sussidio «Verifica in coppia» nel lavoro di squadra e tra squadre non solo risolve problemi di memoria, ma è di grande aiuto per l'acquisizione di abilità comunicative che rompono l'isolamento e la noia dell'esercizio individuale.

\subsection{Verifica in coppia.}

Q uesto sussidio si compone di 8 passi. Può essere usato per un esercizio tradizionale, o per risolvere in modo più creativo un problema posto da una lettura. N el primo caso gli alunni si allenano I'un I'altro in attività di scrittura; mentre nell'altro si allenano per cercare la soluzione al problema posto.

Esaminiamo questo secondo caso, diretto a studenti di livello avanzato. D opo la lettura e analisi di due cataloghi di prodotti uguali, offerti da due aziende distinte, ogni squadra deve decidere quale dei due prodotti è il più conveniente. Comparando prestazioni, prezzi di listino, sconti e tutti gli altri elementi, gli alunni devono completare lo schema seguente:

VANTAGGI

10 prodotto
SVANTAGGI

$2^{\circ}$ prodotto

Un alunno in ogni coppia assume il ruolo dell'allenatore e l'altro cerca di individuare i vantaggi del primo prodotto. L'allenatore aiuta o incoraggia, ma non esegue l'esercizio per l'altro. L'altra coppia fa lo stesso esercizio. Tra le coppie si scambiano i ruoli: adesso l'alunno ex-allenatore cerca i vantaggi del secondo prodotto, mentre il primo alunno, ora allenatore, Io aiuta. L'esercizio continua con scambi di ruoli anche per gli svantaggi del prodotto. Q uando le coppie della squadra hanno finito verificano il risultato dell'esercizio ed eventualmente discutono i punti in disaccordo. Decidono quale prodotto comprare, giustificando la loro scelta attraverso una serie di frasi comparative, secondo degli schemi guidati dall'insegnante (esercizi sui comparativi). L'insegnante ritira gli schemi per correggerli e riconsegnarli alla prossima lezione. Alla lezione seguente, dopo aver riconsegnato i compiti corretti, si propone agli alunni l'analisi e la posteriore scrittura di una lettera di «richiesta di fornitura» alla ditta di cui hanno scelto il prodotto.

\subsection{Le abilità di lettura e scrittura}

L'approccio alla lettura della corrispondenza commerciale, o di qualsiasi testo di economia può essere proposto in due fasi, attraverso sussidi diversi. N e proponiamo due. Per gli alunni principianti eintermedi: la ricostruzione della let- 
tera attraverso l'interazione del lavoro collaborativo. Per l'avanzato, ma anche per gli altri livelli: I'analisi testuale attraverso le tecniche che conosciamo; vale a dire, privilegiando la lettura individuale, prima cursoria e poi di «scanning». Secondo il nostro punto di vista, anche per alcuni esercizi di analisi testuale è possibile un'integrazione tra le due didattiche, quella nozionale-funzionale e quella collaborativa. Infatti, per un economista l'obiettivo principale della lettura è quello di cogliere rapidamente il nucleo centrale delle informazioni, assimilarle per saper condividere il dato nuovo e aggiornato. É su questo punto, sulla tecnica di «skimming» per la lettura, che può rendersi utile una organizzazione collaborativa degli alunni. Le procedure che aiutano la comprensione orale di parti del testo scritto, come quelle di incastro, abbinamento e riordino (Porcelli, 1992), si basano proprio sull'abilità di diffondere l'informazione attraverso la lettura. L'obiettivo per ogni squadra è la ricostruzione di un testo per mezzo di un esercizio orale di «ettura-puzzle». O gni alunno possiede soltanto una parte della lettera commerciale. D eve leggere la sua parte agli altri alunni della squadra che ascoltano in silenzio. Poi ogni membro legge la parte della lettera che gli è stata assegnata e così via, finché tutta la squadra tenta di ricostruire il testo, dandogli un senso compiuto. Se un al unno legge in modo scorretto le frasi assegnategli, difficilmente gli altri capiranno quello che sta dicendo e la squadra non raggiungerà l'obiettivo fissato dall'insegnante. L'alunno mette anche in evidenza la propria capacità di collaborare, con la volontà di articolare bene le parole, per comunicare, responsabilizzarsi a portare a termine insieme agli altri un breve progetto di lavoro.

U na delle abilità sociali che mette a fuoco l'AC è la responsabilizzazione del discente nei confronti del gruppo di lavoro. In questo senso propone, per I'abilità di scrittura, l'attività del «D ettato-puzzle». Attraverso uno scambio di ruoli, tra un alunno che legge e gli altri componenti della sua squadra che scrivono, l'attività coinvolge la capacità dell'alunno di leggere rapidamente, memorizzare dati, riportarli agli altri che devono scriverli. Alla fine la squadra deve verificare il testo ricostruito. A questo punto I'insegnante mostra il testo completo (per es., mediante la lavagna luminosa) e gli alunni correggono gli errori e ne discutono con l'insegnante. $C$ 'è stato qualche conflitto in classe, con questo esercizio, perché mette in evidenza gli alunni con un basso rendimento. Se si introduce un elemento competitivo, attraverso un sistema di punteggi per la verifica del lavoro in classe, a volte una squadra si sente «sabotata» da un suo membro se non riesce a finire l'esercizio e a raggiungere un punteggio soddisfacente.

\section{D irezione, verifica e valutazione}

Durante il corso, per il controllo del raggiungimento degli obiettivi intermedi delle UD, si propongono delle verifiche individuali, ma anche dei test da svolgere in squadra.

Inoltre, alla fine di ogni quadrimestre viene proposta una scheda di autovalutazione globale come squadra. 
Per quanto riguarda l'esame vero e proprio, bisognerebbe riporre in discussione il problema della verifica e della valutazione. $\dot{E}$ impossibile affrontare qui i problemi teorici legati alla pratica della valutazione. Risulta evidente, comunque, che l'esame è proposto in funzione agli obiettivi raggiunti, ma anche dalla linea didattica adottata. La prova d'esame per gli studenti del primo e secondo livello di Italiano commerciale è individuale e consiste in prove di comprensione orale e scritta, seguendo molte delle procedure suggerite da Porcelli (1992). II sistema di verifica dovrebbe tener conto anche del lavoro organizzativo svolto in classe e adottare, quindi, un sistema di punteggio da integrare alla valutazione finale dell'esame. La verifica del gruppo deve tener conto del lavoro d'interazione tra ogni membro di una squadra e considerare anche le attività interattive tra le squadre, con lo scopo di raggiungere un obiettivo comune a tutta la classe.

La fase della programmazione che corrisponde alla definizione degli obiettivi finali può integrarsi con le strategie per raggiungere uno degli obiettivi generali dell'AC, cioè la competenza nel condividere l'informazione tra squadre e con tutta la classe. U no dei contenuti centrali del programma è «L'Azienda», sviluppato attraverso una serie esaustiva di UD. Alla fine del corso avanzato, gli alunni di ogni squadra hanno il compito di presentare in modo esauriente la descrizione specialistica di una «propria» impresa a tutta la classe. Le diverse presentazioni saranno oggetto di verifica e valutazione:

a) da parte dell'insegnante;

b) da parte della classe, mediante apposite griglie. Scegliere per votazione l'impresa migliore del corso.

La classe può valutare: la partecipazione di ogni rappresentante della squadra durante la presentazione; la chiarezza dell'esposizione; la capacità di convinzione; I'originalità e competitività del prodotto, ecc.). Si tratta di una prova finale, a cui si arriva dopo aver superato i molti test delle diverse UD. Un altro aspetto importante è la direzione della classe. N ell'incoraggiare I'interazione comunicativa, si devono usare abilità direttive diverse, per non perdere il controllo della classe. N el suo testo «C ooperative Learning», Spencer Kagan offre ottimi suggerimenti per contrarrestare la tendenza naturale al rumore del lavoro di squadra, oppure per assegnare dei ruoli agli alunni, in modo da responsabilizzarli alla direzione della propria squadra. Infine, un fattore da non sottovalutare èil tempo a disposizione.

\section{Conclusione}

Siccome è fondamental e sviluppare e raggiungere gli obiettivi del programma d'IFS, è necessario integrare gli aspetti interdisciplinari, sforzandosi di adeguarli alle ultime tendenze in glottodidattica, senza escludere l'eredità di nessuno degli approcci sviluppatisi fin'ora. 
Per quanto riguarda l'AC, i protagonisti del processo glottodidattico sono l'individuo (insegnante-apprendente) sempre in rapporto al gruppo e, quest'ultimo, alla classe, come insieme: si tratta di un'esperienza d'apprendimento che si sforza di risolvere l'antinomia «collettivo/individuale», postulata dalla sociolinguistica e oggetto di ricerca anche della scienza cognitiva. T uttavia, I'AC sposta l'obiettivo dal dualismo «docente/discente» per puntarlo sui valori interdipendenti, relativi ai comportamenti determinati dai ruoli sociali tra gli alunni. La sua proposta pedagogica può ai utare il discente ad acquisire le abilità necessarie per una competenza autonoma nella L 2, ma anche per un riutilizzo nella sua pratica professionale.

\section{Bibliografia}

Studi e manuali sull'Apprendimento Cooperativo:

JOHNSON, D.\& R. (1987). Learning together and alone. Englewood Cliffs, N.J.: Prentice $\mathrm{H}$ all.

JOHNSON, D. (1989). Cooperation and Competition. Theory and Research. Edina, M n.: Interaction Book Company.

H IGH , J. (1993). Second Language Learning through Cooperative Learning. San Juan de Capistrano (C alifornia): Kagan Cooperative Learning .

KaGAN, S. (19851) (1993). Cooperative Learning. San Juan de Capistrano (California): Kagan Cooperative Learning.

N un AN, D. (a cura di) (1992). Collaborative Language Learning and T eaching. Cambridge: CUP.

Stevens, R.J.; Slavin, R.E.; Farnish, A.M . (1991). «T he effect of cooperative learning and direct instruction in reading comprehension strategies on main idea identification». J ournal of Educational Psychology, marzo.

Studi sulle LFS, secondo l'approccio comunicativo. N ell'elenco suggeriamo anche alcuni autori che hanno trattato l'apprendimento delle lingue seconde, con una particolare attenzione verso i concetti di obiettivo, bisogno e motivazione.

AA.VV. (1987). Glottodidattica: aspetti e prospettive. Bergamo: Juvenilia.

AA.VV . (1988). Il linguaggio delle scienze e il suo insegnamento. Brescia: La Scuola.

AA.VV. (1990). Le lingue di specializzazione e il loro insegnamento. M ilano: Vita e Pensiero.

BALBONI, P. (1987). «M icrolingue e comunicazione in classe; II professore di microlingue; Parametri per l'analisi dei fattori socio-economici», in AA.VV. 1987.

- (1991). T ecniche didattiche e processi d'apprendimento linguistico. Padova: Liviana.

Caim I ValentINI, A. (1990). «ESP-LSP: un modello teorico-applicativo per una didattica della microlingua», in AA.VV. 1990.

CAMBIAGHI, B. (1990). «Didattica della ricezione del testo microlinguistico», in AA.VV. 1990.

FREDDI, G. (1979). Didattica dellelingue moderne. Bergamo, M inerva I talica.

- (1988). «Linee per una didattica delle microlingue», in AA.VV . 1994.

- (1994). Glottodidattica. T orino: U tet. 
Gard ner, R.C. (1985). Social Psychology and Secon Language Learning. The Role of Attitudes and M otivation. Edward Arnold.

Gardner, R.C.; LAM BeRT, W.E. (1972). Attitudes and M otivation in Second Language Learning. N ewbury H ouse.

G оTTI, M . (1987). «L'insegnamento dei linguaggi specialistici»; «Le attività di lettura e scrittura nella didattica dei linguaggi specialistici»; «Presentazione di una unità didattica per l'insegnamento della lingua straniera nelle scuole ad indirizzo commerciale» in AA.VV. 1987.

- (1991). I linguaggi specialistici. Firenze: La N uova Italia.

H UTCHIN SON; W ATERS (1987). Engl ish for Specific Purposes: a learning-centred approach. Cambridge: CUP.

JULLION, M.C. (1990). «L'insegnamento delle LSP nelle Facoltà di Economia e Commercio, Giurisprudenza e Scienze Politiche. Problemi istituzionali»; «Percorso storico: problemi di didattica della microlingua in tre riviste francesi», in AA.VV. 1990.

M UnBY, J. (1978). Communicative Syllabus D esign. Cambridge: CU P.

PoRCELLI, G. (1987). «I testing in un approccio comunicativo alle lingue estere», in AA.VV. 1987.

- (1990). «D alla lingua comune alle microlingue», in AA.VV . 1990.

- (1992). Educazionelinguistica evalutazione. Bergamo: Liviana.

TITONE, R. (1980). G lottodidattica: un profilo storico. Bergamo: M inerva I talica.

- (19912). La ricerca in psicolinguistica applicata ein glottodidattica. Roma: Bulzoni.

- (1992). G rammatica e gl ottodidattica. N uove prospettive. Roma: Armando.

VogHeRA, M . (1992). Sintassi eintonazione dell'italiano parlato. Bologna: Il M ulino. 